\title{
MICRO- AND PARTIAL GRAVITY ATMOSPHERIC FLIGHT
}

\author{
A.M. (Alwin) Kraeger*, M.M. (René) van Paassen† \\ Delft University of Technology, Faculty of Aerospace Engineering \\ PO box 5058, 2600 GB Delft, The Netherlands \\ E-mail: A.M.Kraeger@lr.tudelft.nl
}

\begin{abstract}
This paper presents a study on a possible control concept for micro- or partial gravity flight of conventional aircraft that can be implemented in any aircraft with a minimum in effort. The method makes use of a conventional pitch rate controller to track the continuously changing pitch rate along the desired micro- or partial gravity trajectory. As a first step towards automatic flight this principle is used to design a micro- and partial gravity flight director. The flight director has been experimentally evaluated in a fixed base manned simulation and its performance checked against other flight director principles. Pilots unanimously pointed out this flight director as their favorite one and it also showed the best performance. A baseline version will be implemented and flight-tested in the Delft University Cessna Citation research aircraft in the near future.
\end{abstract}

\section{Introduction}

In a wide range of scientific disciplines experiments are performed under microgravity conditions to investigate the influence of gravity on systems. These investigations range from physiological experiments on the human body to experiments on the level of molecules in fluids. The required duration of the microgravity periods for these experiments varies from seconds to days or even years. Depending on the duration, some of these experiments can be performed in aircraft in atmospheric flight.

The option of flying partial gravity trajectories, where the specific acceleration lies somewhere in between 0 and 1 times the earth gravitational acceleration, is becoming more and more desired nowadays. It would for example be of interest to simulate the Martian gravitational environment, which is approximately $3 / 8$ times the earth's gravitational field. In principle this should be possible in an aircraft flying a curved trajectory.

In case of microgravity flight the pilot flying the maneuver is able to perform well using only human senses and simple aids, like an accelerometer to show the gravity level. When flying a partial gravity track this may be too much to ask from the pilot. A good and helpful flight director would be beneficial in this case.

In the past a successful attempt has been made by the Delft University of Technology (TUD) to build and fly a microgravity flight director ${ }^{1}$.

\footnotetext{
* PhD candidate, Control \& Simulation Division

$†$ Assistant Professor, Control \& Simulation Division

Copyright (C) 2002 by A.M. Kraeger. Published by the American Institute of Aeronautics and Astronautics, Inc. with permission.
}

With the introduction of a new Flight Test Instrumentation System (FTIS) for the TUD research aircraft, more flexibility in designing and testing of new displays has been created. With the safety pilot in the left seat, the experiment pilot can fly the aircraft from the right seat to evaluate the mounted display. This flexibility is used to design a flight director not only for microgravity flight but partial gravity flight as well.

A control concept is proposed which makes use of the a priori known pitch rate and pitch acceleration during a parabolic or partial gravity trajectory. The concept is illustrated by simulations of partial gravity maneuvers of the Cessna Citation research aircraft.

Different partial gravity flight director designs, based on the proposed control concept but also on more conventional designs, have been implemented and tested in a fixed base simulation in the HumanMachine Interface (HMI) laboratory of the TUD Faculty of Aerospace Engineering.

The most promising concept will be tested in actual flight in the Cessna Citation II laboratory aircraft, which is owned by the TUD Faculty of Aerospace Engineering and the Dutch National Aerospace Laboratory (NLR).

\section{Specific acceleration}

In order to expose a payload to a certain g level, accelerometers placed at the position of the payload should maintain a constant output in three independent directions. The quantity, which has to be controlled, is the specific acceleration. Since the payload, which will be attached to the aircraft is to be exposed to partial gravity, the specific acceleration at the payload must be constant both in magnitude and direction relative to the aircraft's body fixed reference frame. 
To clarify, consider a point mass $\mathrm{m}$ in the gravity field of the earth, subjected to an aerodynamic force $\overline{\mathbf{R}}$, thrust $\overline{\mathbf{T}}$, and gravity force $\overline{\mathbf{F}}_{\mathrm{g}}$. The acceleration of the point mass is:

$\overline{\mathbf{a}}=\frac{\overline{\mathbf{F}}_{t o t}}{m}=\frac{\overline{\mathbf{R}}+\overline{\mathbf{T}}+\overline{\mathbf{F}}_{g}}{m}$

However the output of an accelerometer fixed to the point mass is:

$\overline{\mathbf{a}}^{*}=\frac{\overline{\mathbf{F}}_{t o t}-\overline{\mathbf{F}}_{g}}{m}=\frac{\overline{\mathbf{R}}+\overline{\mathbf{T}}}{m}$

$\overline{\mathbf{a}}^{*}$ is the specific acceleration and $\overline{\mathbf{R}}+\overline{\mathbf{T}}$ is the specific force.

To maintain a constant specific acceleration $\overline{\mathbf{a}}^{*}=\lambda g$ the specific force should be:

$\|\overline{\mathbf{R}}+\overline{\mathbf{T}}\|=\|\overline{\mathbf{L}}+\overline{\mathbf{T}}+\overline{\mathbf{D}}\|=\lambda m g$.

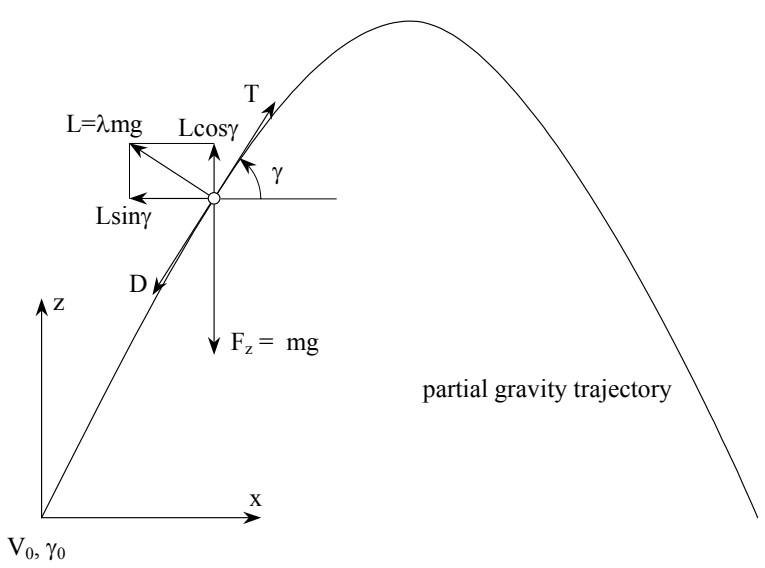

Figure 2.1: The forces on an aircraft during partial gravity flight with specific acceleration $\lambda g$.

As a first approximation it is assumed that thrust acts in the direction of airspeed and cancels drag during the trajectory. In this case only lift generates the specific force, refer to figure 2.1. The main focus will be on the control of the aircraft about the lateral axis by means of the elevator.

In the following a microgravity flight will be considered as a special case of a partial gravity flight. So in this paper the class of flights will be considered where for the specific acceleration $\lambda g$ holds: $0 \leq \lambda g<1$.

\section{The proposed control concept}

\subsection{Introduction}

In order to fly a partial gravity trajectory the forces on the aircraft must be controlled very accurately. To find the required control inputs $\delta_{e}$ (elevator deflection) and $\delta_{t}$ (thrust setting) from the desired specific acceleration, one would need to have an inverse dynamic model ${ }^{2}$ of the aircraft. Often it requires a lot of effort to attain such model and even if it is already available it may not be accurate enough for estimating required control inputs.

The proposed control concept aims at easy implementation in any aircraft and therefore a different strategy has been followed to come to a control concept. A minimum amount of knowledge of the aircraft dynamics is required to implement the proposed flight director or autopilot.

Another important advantage of the concept is that it does not try to follow a certain predefined trajectory. After each perturbation from the nominal trajectory the perturbed aircraft state is taken as a new initial condition for the next nominal trajectory. The partial gravity trajectory may be considered as an infinite number of initial conditions, together making up the trajectory.

One way or the other the forces on the aircraft must remain constant while the airspeed $V$, pitch angle $\theta$ and flight path angle $\gamma$ change according to the required trajectory. This means that for example during microgravity flight the lift on wing and stabilizer must be exactly opposite, while at the same time generating the required moment about the lateral axis to rotate the aircraft to meet the required pitch angle at each instant in time.

In the proposed control concept use is made of the a priori knowledge about the trajectory following an initial condition $\left(V_{0}, \gamma_{0}\right)$. From the initial condition it is possible to calculate the required pitch rate $q$ a time interval $\Delta t$ later. This predicted reference value for $q$, together with the actual value for $q$ can be input to a PID $q$ controller, which gives the required elevator angle to minimize the difference $q_{\text {ref }}-q$.

\subsection{Zero gravity flight}

The equations of motion during an ideal zero $\mathrm{g}$ trajectory (refer to figure 2.1):

$$
\begin{array}{ll}
-m g & =m \ddot{z} \\
0 & =m \ddot{x}
\end{array}
$$

The velocity and position components in a point of a zero $\mathrm{g}$ trajectory following on an initial condition $V=V_{0}$ and $\gamma=\gamma_{0}$ follow from integration: 


$$
\begin{aligned}
& \left\{\begin{array} { l } 
{ \ddot { z } = - g } \\
{ \ddot { x } = 0 }
\end{array} \Rightarrow \left\{\begin{array}{ll}
\dot{z}=-g t+V_{0} \sin \left(\gamma_{0}\right) \\
\dot{x}=V_{0} \cos \left(\gamma_{0}\right)
\end{array} \Rightarrow\right.\right. \\
& \left\{\begin{array}{l}
z=-\frac{1}{2} g t^{2}+V_{0} \sin \left(\gamma_{0}\right) t+h_{0} \\
x=V_{0} \cos \left(\gamma_{0}\right) t
\end{array}\right.
\end{aligned}
$$

The velocity $V$ and flight path angle $\gamma$ during the parabola are found as follows:

$$
\begin{aligned}
V^{2} & =\dot{x}^{2}+\dot{z}^{2}= \\
& =g^{2} t^{2}-2 g V_{0} \sin \left(\gamma_{0}\right) t+V_{0} \sin ^{2}\left(\gamma_{0}\right)+V_{0} \cos ^{2}\left(\gamma_{0}\right)= \\
& =g^{2} t^{2}-2 g V_{0} \sin \left(\gamma_{0}\right) t+V_{0}^{2}
\end{aligned}
$$

$$
\begin{aligned}
\gamma & =\operatorname{arctg}\left(\frac{\dot{z}}{\dot{x}}\right)=\operatorname{arctg}\left(\frac{-g t+V_{0} \sin \left(\gamma_{0}\right)}{V_{0} \cos \left(\gamma_{0}\right)}\right)= \\
& =\operatorname{arctg}\left(\frac{-g t}{V_{0} \cos \left(\gamma_{0}\right)}+\tan \left(\gamma_{0}\right)\right)
\end{aligned}
$$

Taking the first time derivative of $V$ and first and second time derivative of $\gamma$ :

$\dot{V}=\frac{2 g^{2} t-2 g V_{0} \sin \left(\gamma_{0}\right)}{2 V}$

$\dot{\gamma}=\frac{-g V_{0} \cos \left(\gamma_{0}\right)}{g^{2} t^{2}-2 g V_{0} \sin \left(\gamma_{0}\right) t+V_{0}^{2}}$

$\ddot{\gamma}=\frac{-2 g^{3} V_{0} \cos \left(\gamma_{0}\right) t+2 g^{2} V_{0}^{2} \sin \left(\gamma_{0}\right) \cos \left(\gamma_{0}\right)}{-g^{4} t^{4}+4 g^{3} V_{0} \sin \left(\gamma_{0}\right) t^{3}+4 g^{2} V_{0}^{2} \cos ^{2}\left(\gamma_{0}\right) t^{2}-6 g^{2} V_{0}^{2} t^{2}+4 g \sin \left(\gamma_{0}\right) V_{0}^{3} t-V_{0}^{4}}$

It is now possible to calculate $\dot{\gamma}$ and $\dot{V}$ at time $t=0$ where $V=V_{0}$ and $\gamma=\gamma_{0}$.

$$
\begin{aligned}
& \dot{\gamma}(t=0)=\dot{\gamma}_{0}=\frac{-g \cos \left(\gamma_{0}\right)}{V_{0}} \\
& \ddot{\gamma}(t=0)=\ddot{\gamma}_{0}=\frac{-g^{2} \sin \left(2 \gamma_{0}\right)}{V_{0}^{2}} \\
& \dot{V}(t=0)=\dot{V}_{0}=-g \sin \left(\gamma_{0}\right)
\end{aligned}
$$

The pair $\gamma_{0}$ and $V_{0}$ can represent any point of the zero $g$ trajectory, not only the starting point of the parabola. The parabola is considered as a series of initial conditions. Therefore in the following the subscript "0" will be omitted in $V_{0}$ and $\gamma_{0}$.

It is also possible to generate a prediction of $q_{r e f}$ and its derivative in order to compensate for time delays in the system by substituting $t=\Delta t$ in equations (3.5) - (3.7).

\subsection{Partial gravity flight}

In the same way one can derive for the more general case of a partial gravity flight, starting with initial condition $\left\{\mathrm{h}_{0}, \mathrm{~V}_{0}\right\}=\{\mathrm{V} \cos (\gamma), \mathrm{V} \sin (\gamma)\}$ :

$\gamma(t=0)=\operatorname{arctg}\left(\frac{v}{h}\right)$

$\dot{\gamma}(t=0)=\frac{\lambda g-g \cos (\gamma)}{V}$

$\ddot{\gamma}(t=0)=\frac{2 \lambda g \sin (\gamma)-g^{2} \sin (2 \gamma)}{V^{2}}$

Again, it is possible to calculate (3.11) - (3.13) for values of $t=\Delta t \neq 0$, although it requires more calculation effort.

\subsection{The q-controller}

The flight path angle and its first and second time derivative are given by:

$\gamma=\theta-\alpha$

$\dot{\gamma}=\dot{\theta}-\dot{\alpha}=q-\dot{\alpha}$

$\ddot{\gamma}=\dot{q}-\ddot{\alpha}$

Combining this with equations (3-12) and (3-13) the reference pitch rate $q_{r e f}$ and its first time derivative $\dot{q}_{\text {ref }}$ for a partial gravity track are found:

$q_{r e f}=\frac{\lambda g-g \cos (\gamma)}{V}+\dot{\alpha}$

$\dot{q}_{r e f}=\frac{2 \lambda g \sin (\gamma)-g^{2} \sin (2 \gamma)}{V^{2}}+\ddot{\alpha}$

It is assumed that airspeed $\mathrm{V}$ and flight path angle $\gamma$ are continuously measured during flight, however $\dot{\alpha}$ and $\ddot{\alpha}$, which are small quantities, cannot be accurately measured. Instead, the deviation of $n_{z}$ from the desired value is used to correct $q_{r e f}$. When $u, v, w$ are the velocity components along the body fixed reference frame, the acceleration along the $\mathrm{z}$ axis in the center of gravity of the aircraft is given by:

$a_{z}=\dot{w}-q \cdot u$

The first time derivative of the angle of attack can be approximated as:

$\dot{\alpha}=\frac{\dot{w} u-w \dot{u}}{V^{2}} \approx \frac{\dot{w}}{u}$

Assume that $\dot{\alpha}$ and $\ddot{\alpha}$ would be neglected, then the approximated reference pitch rate would be: 
$q_{r e f}^{*}=\frac{\lambda g-g \cos (\gamma)}{V}$

Then,

$a_{z, r e f}-a_{z}^{*}=\dot{w}-\dot{w}-\left(q_{r e f}-q_{r e f}^{*}\right) \cdot u$

$a_{z, r e f}-a_{z}^{*}=-q_{r e f} \cdot u+\frac{\lambda g-g \cos (\gamma)}{V} \cdot u$

$q_{r e f}=\frac{\lambda g-g \cos (\gamma)}{V}+\frac{a_{z}^{*}-a_{z, r e f}}{u}$

So $q_{\text {ref }}$ is now corrected for neglecting $\dot{\alpha}$ by using the error in acceleration $a_{z}$. If some estimate of $\dot{\alpha}$ is available it can be used and it will in the same way be corrected with the error in acceleration $a_{z}$.

The proposed control concept is represented in figure 3.1

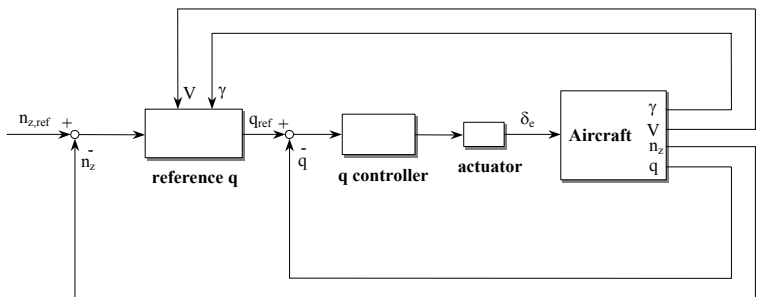

Figure 3.1: The schematic of the control concept

\section{Desktop simulations}

A non-linear model of the Cessna Citation laboratory aircraft was implemented in Matlab/Simulink. Special care was taken to model the short period dynamics correctly. The fidelity of the simulation was checked with flight data obtained from flights where the short period mode oscillation was demonstrated to students by recording the response to an elevator step input.

Figures 4.1 and 4.2 show the measured and simulated aircraft pitch rate and normal specific acceleration responses for an average weight and center of gravity location of the Cessna Citation 550.

Based on the modeled dynamics a PI q controller was designed with proportional feedback gain $4 \mathrm{sec}$ and integrator feedback gain 8 . The reference pitch rate value was calculated from flight path angle, airspeed and normal accelerometer output as described in equation (3-22). It was assumed that the thrust was always counteracting the drag on the aircraft.

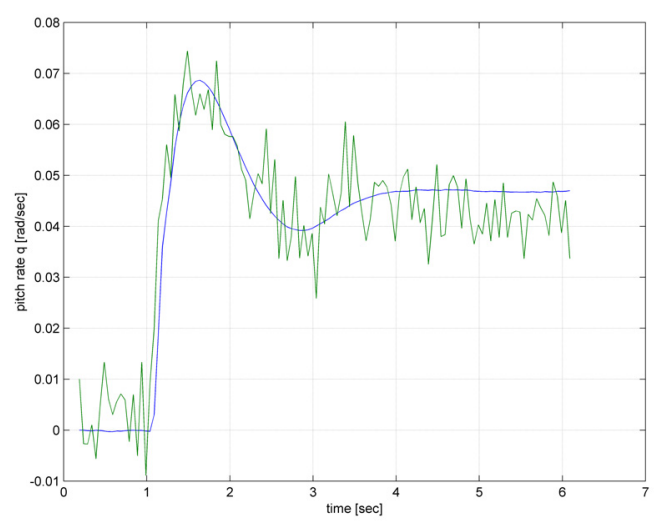

Figure 4.1: Measured and simulated pitch rate response to an elevator step input

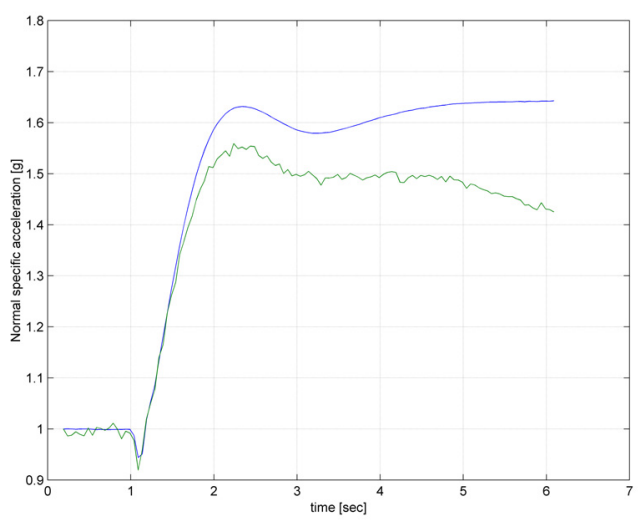

Figure 4.2: Measured and simulated normal specific acceleration response to an elevator step input

\subsection{Microgravity flight}

The results of the simulations for a microgravity and a partial gravity flight with specific acceleration respectively $\lambda g=0$ and $\lambda g=\frac{3}{8} g \quad$ (Martian environment) are shown below. The actual simulated values are in solid lines and the nominal values in dashed lines.

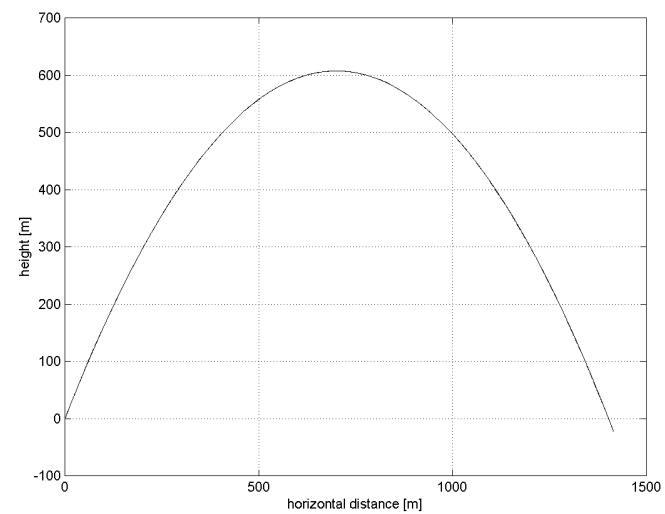

Figure 4-3: The nominal and actual microgravity trajectory 


\subsection{Partial gravity flight}

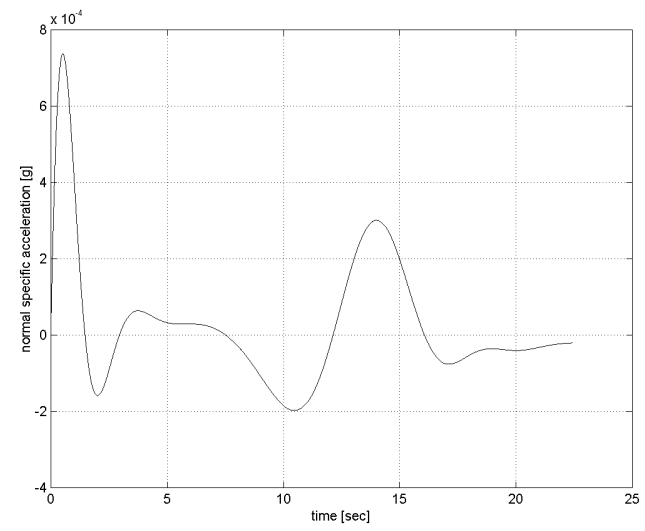

Figure 4-4: The normal specific acceleration during the microgravity trajectory

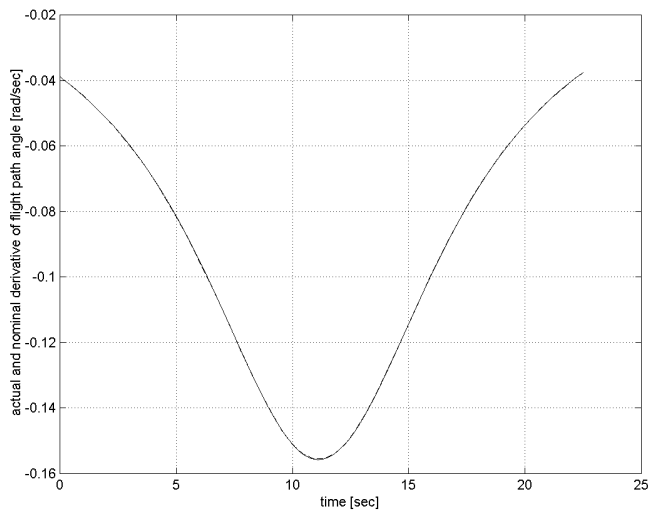

Figure 4-5: The nominal and actual time derivative of the flight path angle $\gamma$ during the microgravity trajectory

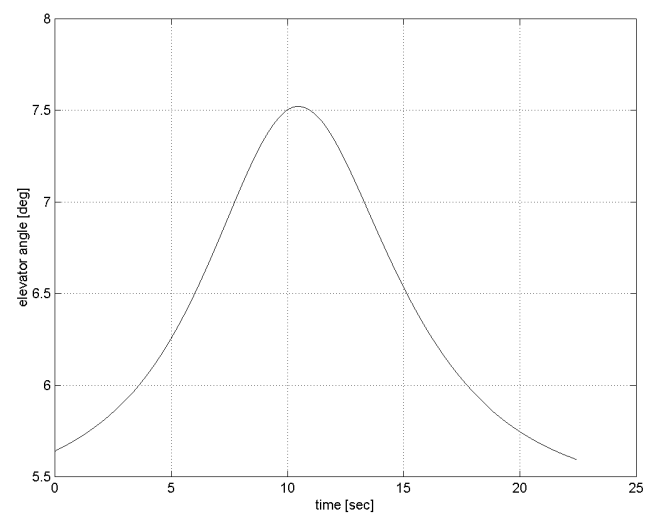

Figure 4-6: The actual elevator deflection $\delta_{e}$ during the microgravity trajectory

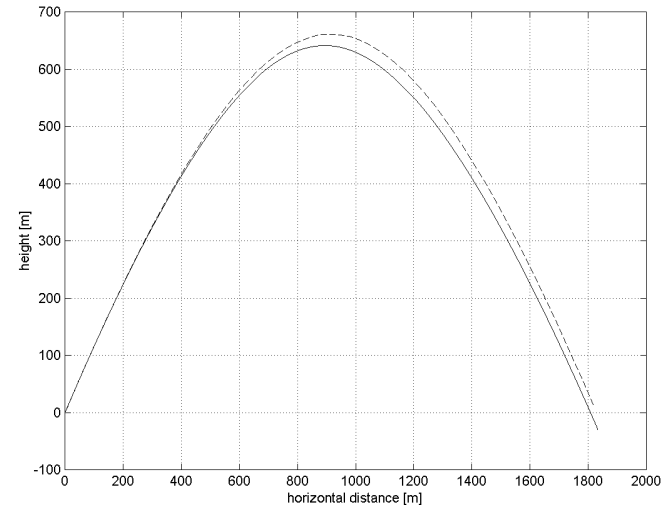

Figure 4-7: The nominal and actual partial gravity trajectory

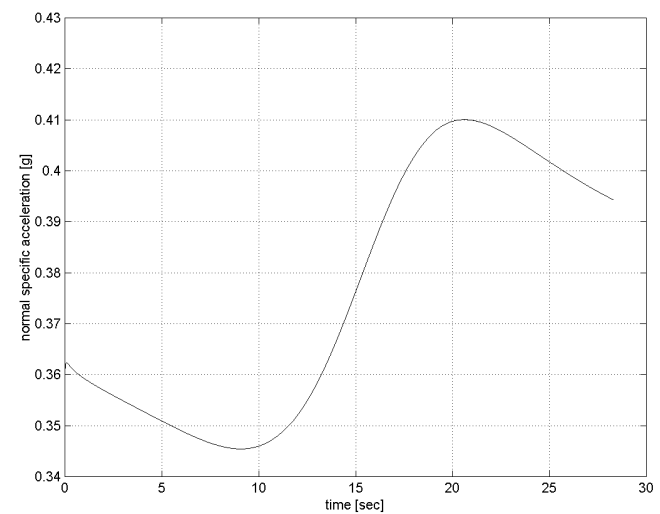

Figure 4-8: The normal specific acceleration during the partial gravity trajectory

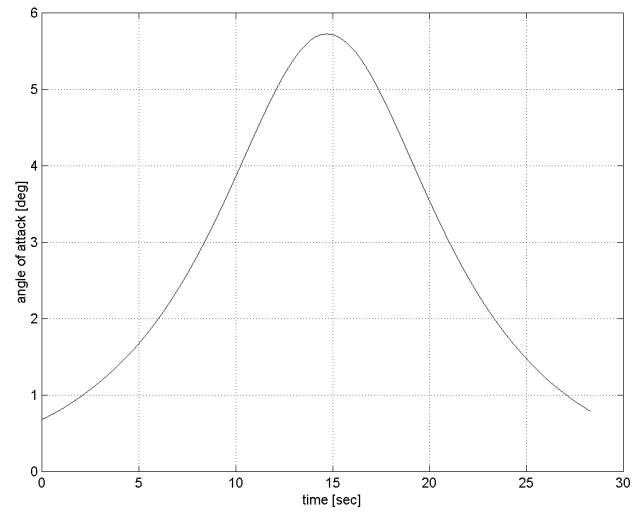

Figure 4-9: The actual angle of attack $\alpha$ during the partial gravity trajectory 


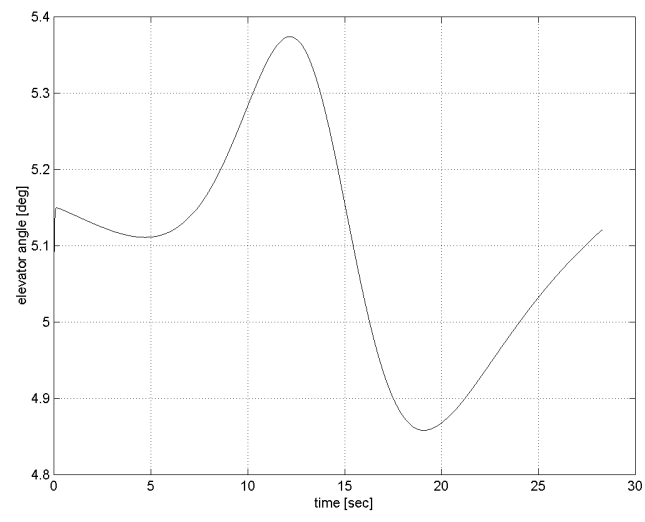

Figure 4-10: The actual elevator angle $\delta_{e}$ during the partial gravity trajectory

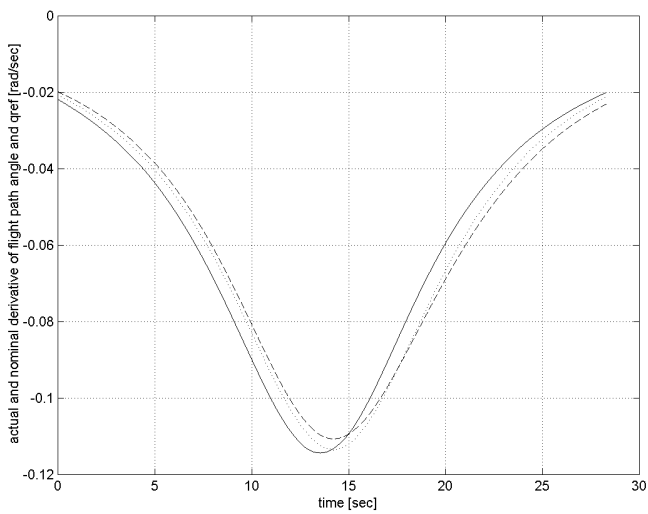

Figure 4-11: Actual (solid) and nominal (dotted) derivative of flight path angle and the value of the calculated $q_{\text {ref }}$ (dashed)

\section{Fixed base manned flight simulation}

The desktop simulations clearly showed that the control concept can be successfully applied to any conventional aircraft when sufficient knowledge of its short period dynamics is available to design a $q$ controller. As a first step towards autoflight the principle was applied to a flight director design. To evaluate its relative performance, different flight director designs were tested in a manned flight simulation experiment.

The experiments were conducted in the Human Machine Interaction (HMI) Laboratory of the Faculty of Aerospace Engineering of the Delft University of Technology. This laboratory consists of a fixed-base flight simulator with two 18" Liquid Crystal Displays (LCD), a hydraulically actuated side-stick and rudder pedals and optionally an outside view projected on the wall of the laboratory.

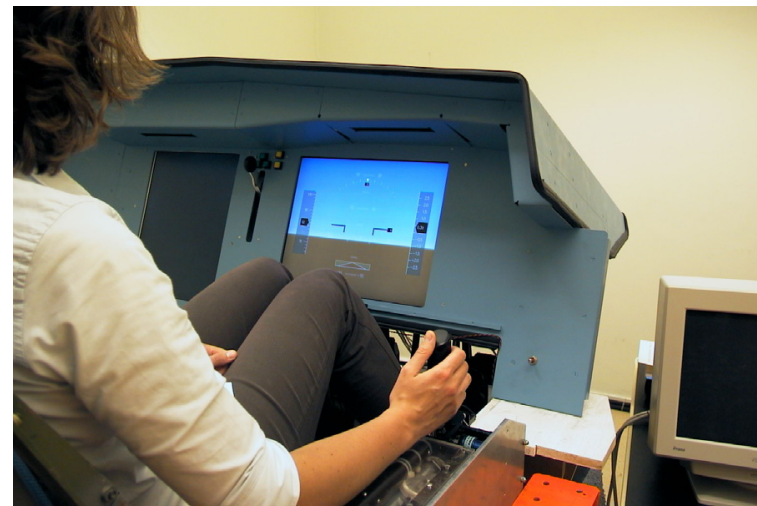

Figure 5.1: Fixed base flight simulator in the HMI lab showing the primary flight display with flight director

For this experiment only one of the LCD displays was used to project a primary flight display with flight director symbols. The aircraft dynamics were simulated using a non-linear model of the Cessna Citation 550, which was frozen in roll and yaw.

The simulation runs under DUECA ${ }^{3}$ (Delft University Environment for Communication and Activation), a middleware layer to facilitate the implementation of simulation models in a distributed real-time simulation environment.

\section{$\underline{\text { 6. Flight director display }}$}

Two different flight director displays were designed, display type 1 and display type 2, as shown in figures 6.1 and 6.2.

Display type 1 shows both a reference value and an actual value of a control signal. A yellow flight director symbol (the upper one in figure 6.1 and 6.2.) represents the desired value and the white symbol (the lower one in figure 6.1 and 6.2) the actual value of the control signal.

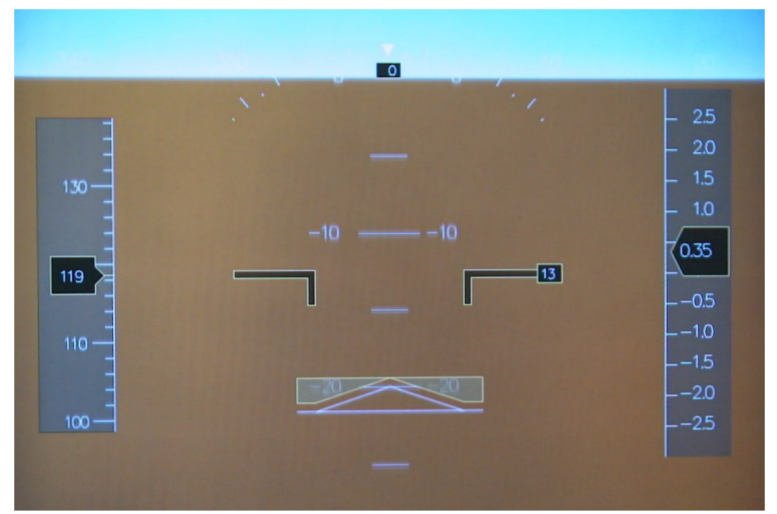

Figure 6.1: Display type 1 
Display type 2 shows only the error signal, meaning that the yellow flight director symbol is fixed to the pitch symbol and only the white symbol is moving.

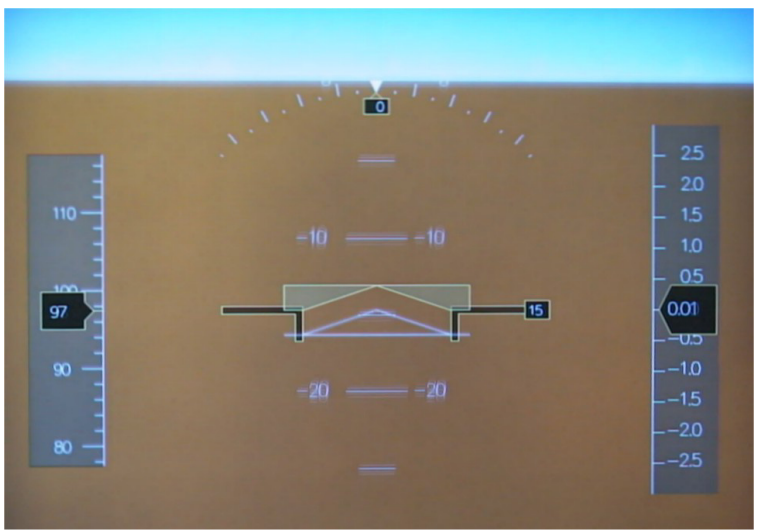

Figure 6.2: Display type 2

The rationale behind display type 1 is that it is supposed to provide feedback to the pilot about the magnitude and rate of change of the desired value of the control signal, by observing the speed with which the yellow symbol is moving away or towards the pitch symbol.

For example, in case the yellow symbol represents the desired pitch rate $q_{\text {ref }}$ and the white symbol represents the actual pitch rate $q$, the distance of the yellow symbol to the pitch symbol is a measure for the magnitude of $q_{\text {ref }}$ and the speed with which the yellow symbol is moving away or towards the pitch symbol is a measure for $\dot{q}_{r e f}$.

The pilot's task is to control the pitch rate $q$ of the aircraft by following the required value. By not only showing the error signal the pilot will have more information to accurately fly the requested trajectory, which by nature requires a non-steady pitch rate.

\section{Tested flight director configurations}

Thirteen different flight director configurations were implemented. They are summarized as EX 1- EX 13 in table 7.1 .

A new flight director is proposed based on tracking of $q_{\text {ref. }}$. This principle is tested with display type 1 (EX 1) and display type 2 (EX 2) to evaluate the influence of the display type. Since in previous work (ref Hosman ${ }^{1}$ ) scaling with dynamic pressure was applied, this will also be tried in this series of experiments (EX 3).

In configuration EX 4 the error signal $q-q_{\text {ref }}$ is filtered to anticipate the short period dynamics. The transfer function for the short period dynamics $H_{\delta_{e}}^{q}(\omega)$ is shown in figure 7.1.

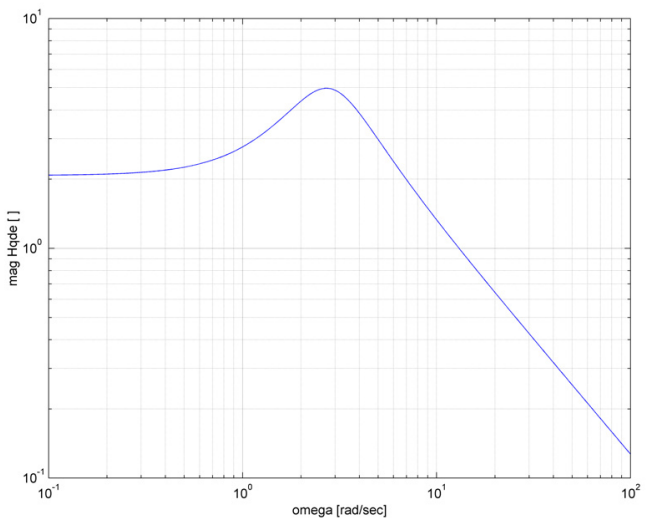

Figure 7.1: The transfer function $H_{\delta_{e}}^{q}(\omega)$

When one filters de error signal $q-q_{\text {ref }}$ with a filter such that the dynamics of the error signal is represented by figure 7.2 , then the pilot can control the normal acceleration by applying a simple gain.

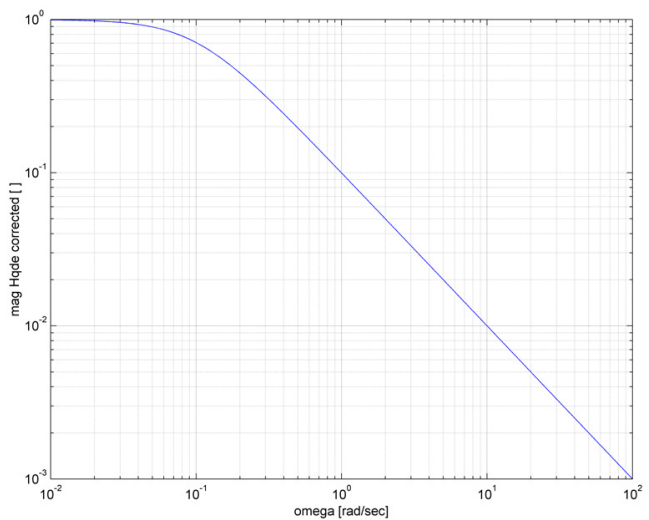

Figure 7.2: The transfer function showing the manipulated dynamics of the displayed signal $q-q_{\text {ref }}$

De rest of the flight director configurations show specific acceleration to the pilot. In configuration EX 5 the specific acceleration error is displayed directly, while in configuration EX 6 it is scaled by dynamic pressure and in configuration EX 7 it is filtered in the same way as described above, but with a different filter. Configurations EX 8 and EX 9 are implementations of the flight director proposed by Hosman $^{1}$ with zero lead and with 0.2 sec lead, respectively. Configurations EX 6 and EX 8 are identical, providing the opportunity to check the consistency of experiment results.

In configurations EX 10 - EX 13 the same PI qcontroller is applied as in the desktop simulations. The display will now show the actual and required elevator deflections or the difference thereof, according to the display type used. In configurations 
11 and 13 feedback of the integrated specific acceleration error is intended to minimize the error in specific acceleration.

\begin{tabular}{|c|l|c|}
\hline EX nr. & Flight director symbol(s) & $\begin{array}{l}\text { Display } \\
\text { type }\end{array}$ \\
\hline 1 & $q$ and $q_{\text {ref }}$ & 1 \\
\hline 2 & $\left(q-q_{\text {ref }}\right)$ & 2 \\
\hline 3 & $\left(q-q_{\text {ref }}\right) / \frac{1}{2} \rho V^{2}$ & 2 \\
\hline 4 & filtered $\left(q-q_{\text {ref }}\right)$ & 2 \\
\hline 5 & $\left(n_{z}-n_{z \text { ref }}\right)$ & 2 \\
\hline 6 & $\left(n_{z}-n_{z \text { ref }}\right) / \frac{1}{2} \rho V^{2}$ & 2 \\
\hline 7 & filtered $\left(n_{z}-n_{z r e f}\right)$ & 2 \\
\hline 8 & $\left(n_{z}-n_{z \text { ref }}\right)_{\text {Hosman }}$ & 1 \\
\hline 9 & $\left(n_{z}-n_{z \text { ref }}\right)_{\text {Hosman }} 0.2$ sec lead & 1 \\
\hline 10 & $\delta_{e}$ and $\delta_{\text {eref }}$ & 2 \\
\hline 11 & $\delta_{e}$ and $\delta_{\text {eref }} ; K n_{z} I=-0.2$ & 2 \\
\hline 12 & $\delta_{e}-\delta_{\text {eref }}$ & 2 \\
\hline 13 & $\delta_{e}-\delta_{\text {eref }} ; K n_{z} I=-0.2$ & 2 \\
\hline
\end{tabular}

Table 7.1: The different flight director configurations (EX nr.) that were tested in the HMI lab

\section{Experiment set-up}

Three test subjects flew 13 different flight director configurations with two different g-conditions, $0.00 \mathrm{~g}$ and $0.38 \mathrm{~g}$, the latter corresponding with the Martian gravitational constant. The $0.38 \mathrm{~g}$ condition was only tested with the first 9 flight director configurations. The pilots flew 5 consecutive maneuvers for each combination of flight director configuration and glevel.

Each maneuver consisted of an acceleration phase where the aircraft was accelerated to 280 KIAS, a pull-up with $2 \mathrm{~g}$, the flight director guidance phase and finally the pull-out with $2 \mathrm{~g}$. The flight director did not provide guidance in the pull-up and the pullout phase. For the $0.00 \mathrm{~g}$ set-point the aircraft was pulled up to $55^{\circ}$ pitch and in case of the $0.38 \mathrm{~g}$ setpoint to $40^{\circ}$ pitch. Thrust was automatically set for zero longitudinal specific acceleration.

\section{Experiment results}

The quality of each maneuver was determined by calculating the mean, the root-mean-square (RMS), the standard deviation, the duration and a quality factor Qtime of the load factor $n_{z}$. These values were determined over the interval where the loadfactor first entered the region within $0.15 \mathrm{~g}$ from the desired value, to the moment the loadfactor left this region at the pull-out. The quality factor Qtime is defined as:

$$
\text { Qtime }=\frac{\text { duration }}{R M S\left(n_{z}\right)}
$$

\subsection{The $0.00 \mathrm{~g}$ condition}

A first overview of pilot performance in the $0.00 \mathrm{~g}$ condition for each of the 13 flight director configurations can be obtained from figures 9.1 and 9.2. These figures show that pilots performed best with configuration 2. Pilot comments also unanimously pointed out configuration 2 as the favorite one.

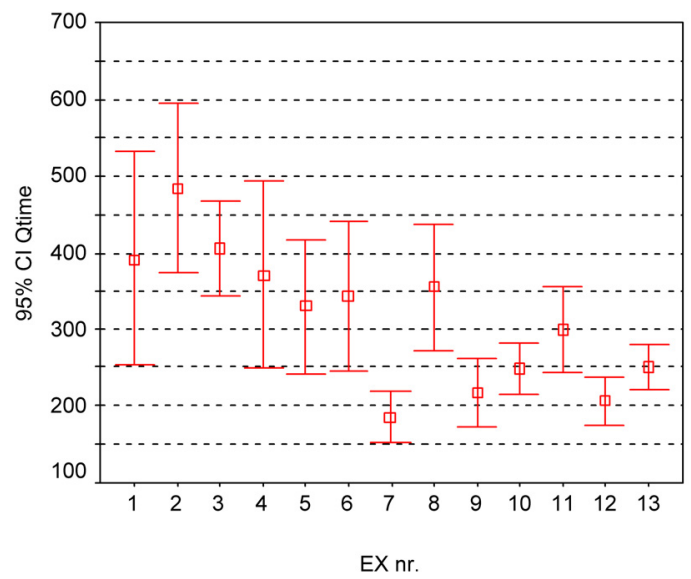

Figure 9.1: Qtime with 95\% confidence intervals for the $0.00 \mathrm{~g}$ condition (higher is better)

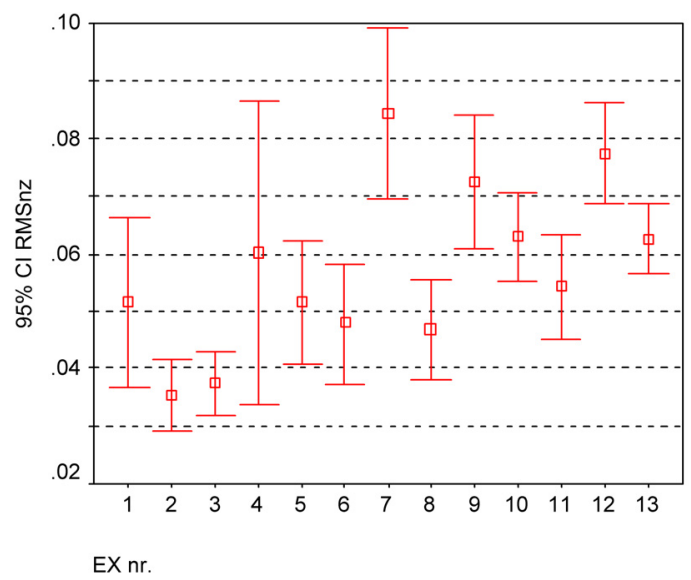

Figure 9.2: $\operatorname{RMS}\left(n_{z}\right)$ with 95\% confidence intervals for the $0.00 \mathrm{~g}$ condition (lower is better)

Table 9.1 shows different subsets of configurations and shows that only subset 1 and 6 are significantly different. 
Means for groups in homogeneous subsets are displayed.

\begin{tabular}{|c|c|c|c|c|c|c|c|}
\hline \multirow{2}{*}{ EX nr. } & \multirow{2}{*}{ Runs } & \multicolumn{6}{|c|}{ Subset for alpha $=.05$} \\
\hline & 1 & 2 & 3 & 4 & 5 & 6 \\
\hline 2 & 15 & .0354 & & & & & \\
\hline 3 & 15 & .0375 & .0375 & & & & \\
\hline 8 & 15 & .0468 & .0468 & .0468 & & & \\
\hline 6 & 15 & .0479 & .0479 & .0479 & & & \\
\hline 5 & 15 & .0515 & .0515 & .0515 & .0515 & & \\
\hline 1 & 15 & .0516 & .0516 & .0516 & .0516 & & \\
\hline 11 & 15 & .0543 & .0543 & .0543 & .0543 & & \\
\hline 4 & 15 & & .0602 & .0602 & .0602 & .0602 & \\
\hline 13 & 15 & & & .0626 & .0626 & .0626 & .0626 \\
\hline 10 & 15 & & & .0629 & .0629 & .0629 & .0629 \\
\hline 9 & 15 & & & & .0725 & .0725 & .0725 \\
\hline 12 & 15 & & & & & .0775 & .0775 \\
\hline 7 & 15 & & & & & & .0844 \\
\hline Sig. & & .217 & .071 & .465 & .120 & .195 & .052 \\
\hline
\end{tabular}

Table 9.1: Homogeneous subsets RMS $\left(n_{z}\right)$ - StudentNewman-Keuls

Figure 9.3 shows the RMS values of $n_{z}$ for the $0.00 \mathrm{~g}$ condition per pilot.

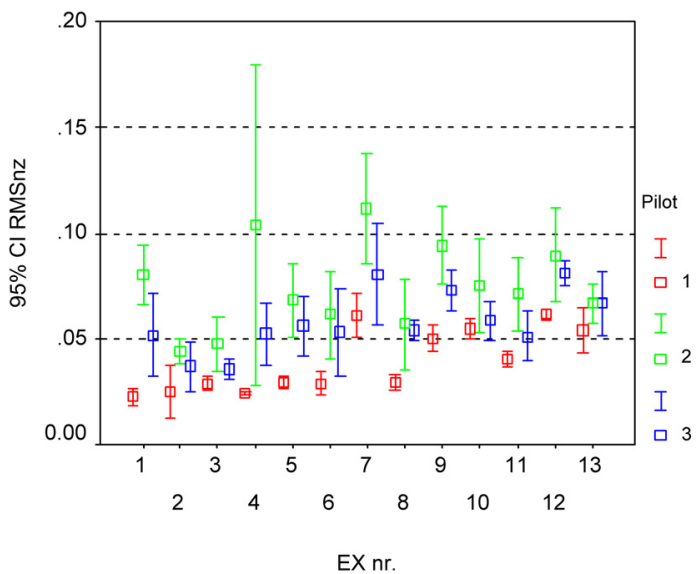

Figure 9.3: $\operatorname{RMS}\left(n_{z}\right)$ with 95\% confidence intervals for the $0.00 \mathrm{~g}$ condition per pilot

Pilots 2 and 3 indicated that they disliked display type 1 and preferred display type 2 . The moving flight director symbols caused confusion and did not contribute to pitch rate and pitch acceleration awareness. The main reason for this is the pitch ladder, which also moves relative to the pitch symbol.

It appears that gain scheduling the displayed error signal by dividing it by dynamic pressure, does not significantly alter the pilot performance. In case of displaying $\left(n_{z}-n_{z \text { ref }}\right)$ it seems to help, but in case of displaying the $\left(q-q_{\text {ref }}\right)$ signal it seems to have an adverse effect.

The classical way of designing a flight director by pre-filtering the error signal to anticipate aircraft dynamics ${ }^{4}$, does not seem to lead to good results in the case of a partial gravity flight director.

The problem is that past performance influences the actual displayed error signal. The flight director will compensate a positive deviation from the desired glevel in the past with a negative deviation in the future, causing only correct guidance in the long run when no more mistakes are made.

Configuration 7 forces the pilot to fly the parabola that follows from the initial condition, which existed at the time the flight director, became active. Each deviation from this parabola leads to another deviation from the desired g-level in order to return to the original parabola. In the same situation in configuration $4, q_{\text {ref }}$ will be adjusted to a new initial condition, explaining the better performance of this configuration.

An extra handicap of configuration 4 and 7 was that the flight director only became active when the actual g-level was within $0.15 \mathrm{~g}$ from the desired g-level, causing an unavoidable transient effect. Pilot 1, who received most training, shows better results with configuration 4 .

Configuration 8 , the flight director principle as proposed by Hosman ${ }^{1}$ with zero lead is exactly the same as configuration 6 . The Hosman configuration with $0.2 \mathrm{sec}$ lead seems to perform worse than the configuration without lead-time, but it must be remarked that the $0.2 \mathrm{sec}$ lead was found optimal during flight tests in the real aircraft. These conditions do not compare with those of the fixed base simulations in the laboratory.

\subsection{The $0.38 \mathrm{~g}$ condition}

Figure 9.4 shows the pilot performance using the first nine configurations for the $0.00 \mathrm{~g}$ and the $0.38 \mathrm{~g}$ condition. Performance in terms of RMS value of $n_{z}$ is higher than in the $0.00 \mathrm{~g}$ condition, probably because of the smoother trajectory.

Figure 9.5 shows the results in terms of $\operatorname{RMS}\left(\mathrm{n}_{\mathrm{z}}\right)$ for the $0.38 \mathrm{~g}$ condition per pilot. More or less the same trend can be observed for all pilots, with an exception for configuration EX 1 for pilot 1.

In the $0.38 \mathrm{~g}$ case configuration 3 seems to be best performing, although it is close to configuration 2 . Scaling the displayed error signal with dynamic pressure has a positive influence in the $0.38 \mathrm{~g}$ case, although the results found in this experiment are not significant. 


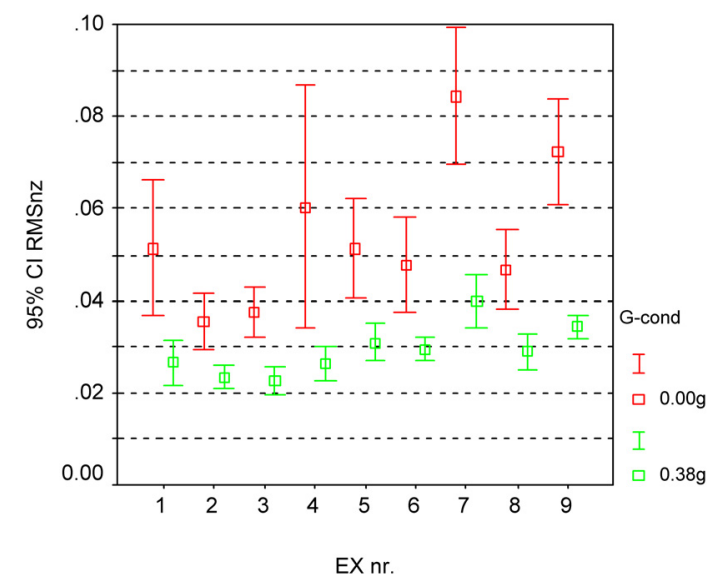

Figure 9.4: $\operatorname{RMS}\left(n_{z}\right)$ with 95\% confidence intervals for the $0.38 \mathrm{~g}$ condition in comparison with the $0.00 \mathrm{~g}$ condition

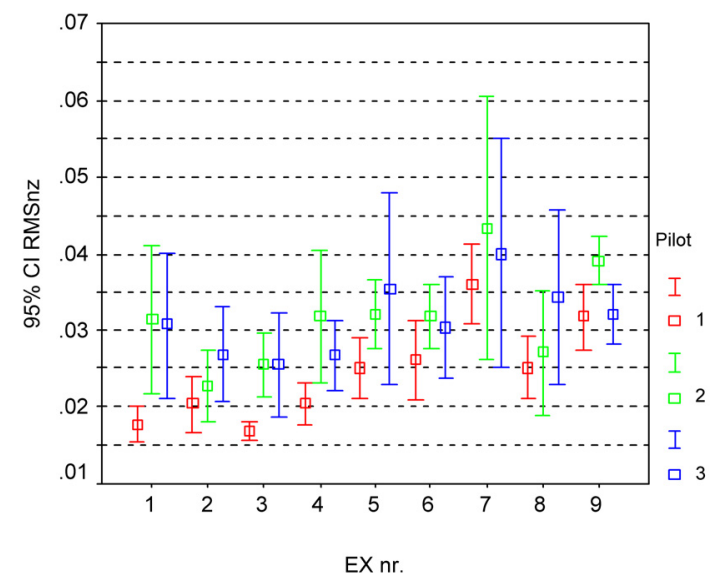

Figure 9.5: $\operatorname{RMS}\left(n_{z}\right)$ with 95\% confidence intervals for the $0.38 \mathrm{~g}$ condition per pilot

\subsection{Effect of generating a prediction}

The most promising configuration (EX 2) was used for further optimization by predicting $q_{\text {ref }}$ some time ahead. Calculating a prediction for $q_{r e f}$ is easy as is explained in section 3.2.

Figures 9.6 and 9.7 show the results of this experiment.

Unfortunately, no significant results can be deduced from these figures. The trend seems to be a decreasing quality of the maneuver with increasing prediction time, although possibly a small benefit can be expected from small prediction times in the order of $0.05 \mathrm{sec}$.

Although performance did not improve in the fixed base simulation, it is very well possible that in real flight a prediction does have a benefit, because it can compensate for inherent time delays in the aircraft instrumentation that are absent in the ideal circumstances of a simulation environment.

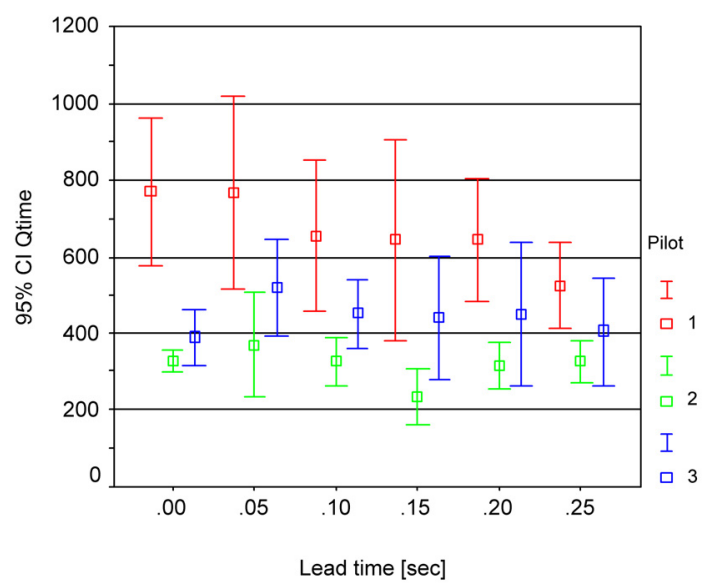

Figure 9.6: $\operatorname{RMS}\left(n_{z}\right)$ with 95\% confidence intervals for EX $n r .2$ in the $0.00 \mathrm{~g}$ condition with increasing prediction time

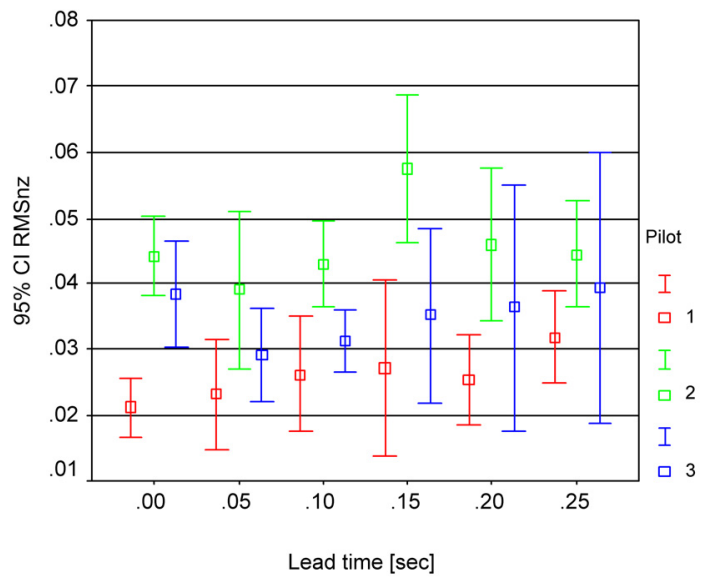

Figure 9.7: $\operatorname{RMS}\left(n_{z}\right)$ with 95\% confidence intervals for EX nr. 2 in the $0.00 \mathrm{~g}$ condition with increasing prediction time, shown per pilot

\section{$\underline{\text { 10. Conclusions }}$}

The control concept that was introduced offers the advantage of easy implementation and requires little knowledge of the aircraft dynamics. Only longitudinal and normal specific acceleration, pitch angle $\theta$, pitch rate $q$, angle of attack $\alpha$ and true airspeed $V$ are required to calculate the reference pitch rate $q_{\text {ref }}$. Some knowledge about the short period dynamics of the aircraft helps in designing a stable and effective pitch rate controller to track $q_{\text {ref }}$.

The same control concept enables partial gravity flight where the specific acceleration lies somewhere in between 0 and $1 \mathrm{~g}$.

It is easy to generate predictions of the reference pitch rate to compensate for time delays in the system. As opposed to direct control of the normal specific acceleration no specific knowledge of aircraft dynamics is required to generate a prediction. 
Manned flight simulation showed that the control concept applied to a flight director gives the best pilot performance, both in a $0.00 \mathrm{~g}$ and a $0.38 \mathrm{~g}$ condition.

\section{Future work}

The flight director will be installed in the Cessna Citation II research aircraft and tested in actual flight. An optimal prediction time will be determined to compensate for system delays and guidance will be extended to entry and recovery of the maneuver. In a later stage it will be attempted to use the elevator trim tab servo to control the normal specific acceleration during automatic partial gravity flight.

\section{$\underline{\text { Acknowledgements }}$}

I would like to thank the test pilots Hessel Benedictus, retired captain B-747 at Royal Dutch Airlines (KLM) and TUD research pilot, and Koen van Engelen, copilot B-747 at KLM for their cooperation in the experiment and their useful comments.

\section{List of symbols}

$\begin{array}{ll}\overline{\mathbf{a}} & \text { acceleration } \\ \overline{\mathbf{a}}^{*} & \text { specific acceleration } \\ \overline{\mathbf{D}} & \text { drag } \\ \overline{\mathbf{F}}_{\mathrm{g}} & \text { gravity force } \\ \overline{\mathbf{F}}_{t o t} & \text { total force } \\ g & \text { Earth's gravitational constant } \\ h & \text { horizontal velocity } \\ K n_{z} I & \text { feedback gain of the integrated specific } \\ & \text { acceleration error } \\ \overline{\mathbf{L}} & \text { lift } \\ m & \text { mass } \\ n_{z} & \text { normal load factor } \\ q & \text { pitch rate } \\ q_{r e f} & \text { reference or desired pitch rate } \\ \overline{\mathbf{R}} & \text { total aerodynamic force } \\ t & \text { time } \\ \overline{\mathbf{T}} & \text { thrust } \\ v & \text { vertical velocity } \\ V & \text { true airspeed } \\ x & \text { horizontal position } \\ z & \text { vertical position } \\ \alpha & \text { angle of attack } \\ \gamma & \text { flight path angle } \\ \delta & \text { elevator deflection } \\ \lambda & \text { specific acceleration expressed in g } \\ \theta & \text { pitch angle } \\ & \end{array}$

\section{$\underline{\text { References }}$}

1. R.J.A.W. Hosman, Flight Director Guidance throughout the Parabolic Maneuver, 1999 IEEE

2. F. Mora-Camino and A.K. Achaibou, ZeroGravity Atmospheric Flight by Robust Nonlinear Inverse Dynamics, Journal of Guidance, Vol. 16 No 3: Engineering notes

3. M.M. van Paassen, O. Stroosma and J. Delatour, DUECA - Data-driven Activation in Distributed Real-time Computation, 2000 AIAA

4. Klein R.H., D.T. Mc Ruer and D.H. Weir, $A$ Pilot-Vehicle Approach to Longitudinal Flight Director design, Proceedings of the Sixth Annual Conference on Manual Control. Wright Patterson AFB, Ohio, April 7-9, 1970.

bold and overlined symbols are vectors. 